\title{
Clinical significance of plasma PAF acetylhydrolase activity measurements as a biomarker of anaphylaxis: cross-sectional study
}

\author{
Krzysztof Piwowarek ${ }^{1}$, Agnieszka Rzeszotarska ${ }^{1}$, Jolanta Korsak ${ }^{1}$, Aleksandra Juszkiewicz ${ }^{1}$, \\ Andrzej Chciałowski ${ }^{1}$, and Jerzy Kruszewski ${ }^{1}$ \\ ${ }^{1}$ Military Institute of Medicine
}

October 12,2020

\begin{abstract}
Background: Platelet-activating factor (PAF) has a direct role as a mediator in the pathogenesis of various disorders with an inflammatory component, including those with allergic aetiology. The peripheral blood concentration of PAF is dynamically regulated by plasma PAF acetylhydrolase (PAF-AH). Previous studies suggest that low activity of plasma PAF-AH could be a predictive marker for increased severity of some types of allergic hypersensitivity reactions - especially anaphylaxis. Aim of the study: The purpose of the study was to evaluate the association between plasma PAF-AH activity and severity in patients with anaphylactic reactions following a wasp or bee sting. Methods: The study group of 89 patients was divided into two subgroups depending on the increasing severity of the most severe anaphylactic reaction in the past, which was assessed according to the Müller's scale. A control group of 20 people was established. Plasma PAF-AH activity was measured using a colorimetric method. Results: It has been observed that plasma activity of platelet-activating factor acetylhydrolase was significantly lower in patients with anaphylaxis history compared to the control group with negative atopic history (on average $21.38 \mathrm{nmol} / \mathrm{min} / \mathrm{ml}$ for the control group, $9.47 \mathrm{nmol} / \mathrm{min} / \mathrm{ml}$ for the first subgroup and $10.16 \mathrm{nmol} / \mathrm{min} / \mathrm{ml}$ for the second subgroup, in both cases $\mathrm{p}<0.0001)$. Conclusion: The plasma activity of PAF-AH is a promising parameter that can help to distinguish a group of patients not threatened with development of anaphylaxis and not requiring laborious or expensive prophylactic procedures.
\end{abstract}

\section{1 | INTRODUCTION}

Platelet-activating factor (PAF), which was discovered in 1972 [1], is considered an important hypersensitivity mediator. Its influence includes anaphylaxis and its most severe form - anaphylactic shock. In the murine $\mathrm{BALB} / \mathrm{c}$ model it was found, that PAF together with histamine may be responsible for the occurrence of anaphylaxis. The effect of PAF was more related to the reduction of cardiac output than to generalized vasodilation [2]. Moreover, some reports suggest that the importance of PAF as a mediator of anaphylaxis in humans may be superior to histamine and tryptase [3].

The concentration of PAF in the peripheral blood is regulated by plasma PAF acetylhydrolase (PAF-AH) [4, 5]. The physiological influence on this enzyme seems to be multidirectional $[6,7]$. Previous studies indicate that the reduced activity of PAF-AH may be a potential risk factor not only for the occurrence but also for the severe course of food and Hymenoptera venom allergies (HVA) [8-10].

The aim of the study was to assess the relationship between the plasma PAF-AH activity and the severity of anaphylactic reaction after honeybee and wasp sting, towards a preliminary analysis of using this relationship for diagnostic purposes.

\section{2 | METHODS}




\section{1 | Setting, study population, inclusion and exclusion criteria}

The cross-sectional study was conducted in the Department of Infectious Diseases and Allergology and in the outpatient allergology clinic of the Military Institute of Medicine in Warsaw from December 2017 to April 2019 .

All study participants had to be of legal age. The study group included patients who registered in the clinic due to an anaphylactic episode of varying severity after being stung by a honey bee or wasp. The episode had occurred at least 21 days before enrolment in the study. The control group consisted of healthy people with negative atopic history. The exclusion criteria for both groups were identified as below:

1. The level of tryptase in the peripheral blood serum over $20 \mathrm{ng} / \mathrm{ml}$.

2. History of congenital dyslipidemia.

3. History of mast cell dysfunction.

4. Acute disease.

\subsection{1 | Patients and controls}

A group of 101 patients with confirmed IgE-dependent HVA were qualified to participate in the study. Subsequently, 6 persons were disqualified due to the presence of exclusion criteria.

The final study group of 45 women and 44 men, was then divided into two subgroups: I-II, including participants with a history of hypersensitivity reactions of maximum severity up to the second degree according to the Müller's scale [11] and III-IV, consisting of patients who have experienced at least one grade III or IV reactions in the past.

9 women and 11 men were qualified for the control group. They were healthy and in a similar age range to the study group. They presented a negative history of allergic and non-allergic hypersensitivity reactions and did not meet the exclusion criteria.

\section{3 | Ethical issues}

The study was performed according to the principles of the ICH-GCP consolidated guidelines and the ethical principles regarding the current revision of the Declaration of Helsinki together with Polish legal and regulatory requirements [12]. Military Institute of Medicine Bioethics Commission approval was obtained (number of decision: 58/WIM/2016).

\section{4 | Data collection}

After obtaining informed consent, the researcher interviewed the participants with a uniform questionnaire comprising of demographic and other data necessary for proper group qualification. Subsequently, basic anthropometric measurements were performed. Serum tryptase levels were determined using the ImmunoCAP method.

Blood samples for the measurement of plasma PAF-AH activity were collected on citrate and transported on ice to the laboratory. Afterwards, the samples were centrifuged at $4 \operatorname{degC}$ for 10 minutes at an acceleration of 700 to $1000 \mathrm{~g}$. The obtained plasma was stored frozen at $-80 \mathrm{degC}$. Finally, the authorized laboratory analyst defrosted samples and loaded into the PAF Acetylhydrolase Assay Kit item No. 760901 produced by Cayman Chemical Company (USA). The test, based on the colorimetric method, was performed according to the manufacturer's instructions. We used radiation with a wavelength of $412 \mathrm{~nm}$. Measurements of the absorbance were made six times in a row, at 1-minute intervals in order to plot the appropriate linear function. The plasma PAF-AH activity was calculated in units of $\mathrm{nmol} / \mathrm{min} / \mathrm{ml}$ using a properly modified formula provided by the manufacturer of the kit.

\section{5 | Statistical analysis}


All statistical tests were performed using the STATISTICA 13.0 software provided by StatSoft Inc. (Poland). The Kolmogorov-Smirnow test was used to determine the significance of differences in the distribution of the studied variables in relation to the normal distribution. The t-test was used for the comparison of means from the groups with a normal distribution, while for others the Mann-Whitney U test was used. For comparisons of multiple groups, the Bonferroni correction for the t-test and Kruskal-Wallis ANOVA rank test were used, respectively.

Categorical variables were presented as n (\%) and compared with each other using the $\chi^{2}$ test. The control group and research subgroups were paired to test the null hypothesis that plasma PAF-AH activity is not related to the severity of the post-stinging reaction. The established level of statistical significance was $\alpha=$ 0.05 .

The correlations between the variables were investigated by calculating the Spearman's rank coefficient. It was considered significant when: $r>0.3$ or $r<-0.3$.

A regression analysis was performed and the receiver operating characteristic (ROC) curve was laid out. Typical test parameters were calculated according to well-known definitions, such as sensitivity, specificity, accuracy, positive predictive value and negative predictive value [13].

\section{3 | RESULTS}

\section{1 | Population characteristics}

As shown in table 1 and 2, subgroups I-II included 8 patients with a history of the first-degree reaction and 31 patients after second-degree reactions. Subgroups III-IV included 33 patients after third-degree reactions and 17 patients after fourth-degree reactions, respectively.

No statistically significant differences were found between subgroups I-II, III-IV and the control group in terms of basic demographic, anthropometric and biochemical data. In the studied subgroups, IgE-dependent reaction to wasp venom was a more frequent cause of post-stinging reactions $(84.62 \%$ in subgroups I-II and $80 \%$ in subgroups III-IV, $\mathrm{p}=0.98)$ than allergy to honey bee venom $(15.38 \%$ in groups I-II and $20 \%$ in groups III-IV, $\mathrm{p}=0.49)$; however, these differences were statistically insignificant.

\section{2 | Plasma PAF-AH activity}

The distributions of PAF-AH activity in the study group and the control group were presented in figure 1. They differed substantially, which was reflected in the highly statistically significant difference in the arithmetic means in both groups.

As shown in figure 2, no significant differences were found in the arithmetic mean values of plasma PAF$\mathrm{AH}$ activity in the individual degrees of severity of anaphylaxis $(\mathrm{p}>0.05)$. Although, these values were considerably lower than the value in the control group, which was $21.38 \pm 9.50 \mathrm{nmol} / \mathrm{min} / \mathrm{ml}(\mathrm{p}<0.0001)$.

According to table 2, the arithmetic mean values of plasma PAF-AH activity in the subgroup I-II (9.47 +- $1.87 \mathrm{nmol} / \mathrm{min} / \mathrm{ml})$ and in the subgroup III-IV $(10.16+-2.50 \mathrm{nmol} / \mathrm{min} / \mathrm{ml})$ also did not differ significantly $(\mathrm{p}>0.05)$, but were substantially lower as compared to the value in the control group $(\mathrm{p}<$ $0.0001)$.

The arithmetic mean values of plasma PAF-AH activity in the smokers and non-smokers did not differ significantly $(9.46+-1.95 \mathrm{nmol} / \mathrm{min} / \mathrm{ml}$ vs $10.13+-1.61 \mathrm{nmol} / \mathrm{min} / \mathrm{ml}, \mathrm{p}=0.201)$.

There was no statistically significant correlation between the plasma PAF-AH activity and most of the parameters, including the age of the patients $(\mathrm{r}=-0.035)$. There was only a statistically significant, but clinically irrelevant, correlation with tryptase concentration $(\mathrm{r}=0.21)$.

\subsection{The performance of PAF-AH activity measurements in recognizing anaphylaxis}

The result of the regression analysis is shown in figure 3. The optimal cut-off point for PAF-AH activity to discriminate anaphylactic and non-anaphylactic patients was $17.24 \mathrm{nmol} / \mathrm{min} / \mathrm{ml}$ at the following parameters: 
- Sensitivity: $100 \%$

- Specificity: $60 \%$

- Accuracy: $93 \%$

- Positive predictive value (PPV): $92.2 \%$

- Negative predictive value (NPV): $100 \%$

- Misclassification rate: $7 \%$

- Area under the curve (AUC): 0.854

- Slope factor: 0.21

\section{4 | DISCUSSION}

In the presented study it was confirmed that the mean activity of plasma PAF-AH activity is significantly lower in patients with a history of anaphylaxis in the course of HVA as compared to healthy people. It confirms the vital role of PAF in the pathogenesis of anaphylactic reactions. Assuming that the plasma PAF-AH activity illustrates the systemic activity of this enzyme, this fact indicates that apart from external factors, the individual's specific predisposition resulting from the enzyme activity may also play an important role.

Contrary to other authors, our research did not show an obvious correlation between PAF-AH activity and the severity of the hypersensitivity reaction to the venom [10]. This problem has been studied and discussed since the early 2000s. Vadas et al. analyzed the relationship between plasma PAF-AH activity and the severity of anaphylactic reaction after peanut consumption in children $[8-9,14]$. In the prospective part of the mentioned authors study, three groups with increasing severity of anaphylaxis were recruited. The control was a group of non-allergic children. In contrast to our study, it showed a trend suggesting that decreased PAF-AH activity is associated with a more severe course of an anaphylactic reaction. However, statistical significance was not obtained.

A statistically significant association was found in the retrospective section of the study. It compared 9 cases of fatal peanut anaphylactic reactions to several other groups, including children who died from non-anaphylactic causes, non-allergic children, and children with urticaria or peanut-induced angioedema. In all these comparisons, the plasma PAF-AH activity was significantly lower in children who died from anaphylaxis. The study did not show any differences in PAF-AH activity in groups of children with bronchial asthma of varying severity $[8,9]$.

In subsequent years, the same researchers found that patients with the lowest levels of plasma PAF-AH activity had a 27-fold higher risk of severe or fatal anaphylaxis. Moreover, the plasma activity of the enzyme was strongly correlated with the concentration of apolipoprotein B. It is noteworthy that the activity of $\mathrm{PAF}-\mathrm{AH}$ is also correlated with the concentration of LDL and decreases during treatment with lovastatin or fenofibrate. Therefore, it cannot be ruled out that lipid-lowering therapy and lowering PAF-AH activity may lead to the increased risk of a severe anaphylactic reaction in a susceptible population [14]. Finally, Vadas et al. found a correlation between PAF-AH activity and the age of the participants, but in our study, it was lacking.

Research on the plasma PAF-AH and food anaphylaxis was also conducted in the Moscow study groups of children with a history of food anaphylaxis, atopic dermatitis and a negative history of allergic diseases. It was found that children with a history of food anaphylaxis had statistically lowered level of PAF-AH mRNA in peripheral blood compared to both other groups of children. In the group with atopic dermatitis, the difference was more than three times. In the group of children with a history of food anaphylaxis, lower expression of the PAF-AH gene was associated with a more severe course of anaphylaxis and cardiovascular symptoms [15].

A large study on the relationship between plasma PAF-AH activity and the severity of anaphylactic reactions to Hymenoptera venom was carried out in a Milan (Italy). Its structure was relatively similar to our trial. There was no statistically significant difference in PAF-AH activity between healthy controls and patients after the mild anaphylactic reactions of the $1^{\text {st }}$ degree of severity. The subgroups with higher severity grades 
had significantly and stepwise lower mean plasma PAF-AH activity compared to the control group and to each other. The exception has occurred for the 3rd and 4th severity subgroups, between which the difference was statistically irrelevant. Logistic regression showed that only PAF-AH activity was significantly associated with the severity of anaphylaxis. The best cut-off point for grades II-IV was $25 \mathrm{nmol} / \mathrm{ml} / \mathrm{min}$ [10].

The outcomes of our trial differ from the results of the presented studies. The difference in the mean activity of the enzyme between the control group and the study subgroups was more than twofold. The highest PAF-AH activity in a patient with a positive history of anaphylaxis was only $17.24 \mathrm{nmol} / \mathrm{min} / \mathrm{ml}$. The cut-off point of $20 \mathrm{nmol} / \mathrm{min} / \mathrm{mL}$ proposed by Pravettoni et al. discriminates patients who are not at risk of developing severe anaphylaxis very well, but our study suggests the possibility of further lowering this threshold. Notwithstanding, the most intriguing is the combination of two study subgroups - grade I-II and III-IV. They did not differ in terms of enzyme activity $(9.47 \mathrm{vs} 10.16 \mathrm{nmol} / \mathrm{min} / \mathrm{ml}, \mathrm{p}=0.0609$ in Student's t-test). Therefore, we did not find a relationship between the severity of anaphylactic reaction in the past and the plasma PAF-AH activity. This is interesting in the context of the studies by Pravettoni et al., which show this kind of association.

There are several potential reasons for the above-mentioned divergence. Apart from the various size of the samples, age of the participants and distinct local practice in qualifying patients for the different severity grades of anaphylaxis, the discussed studies differed in design. Unlike the first Vadas studies, it seems that our study group comprised patients with less severe reactions, e.g. we did not investigate cases of fatal anaphylaxis. The study performed by Pravettoni et al. concerned only newly diagnosed patients, while our study did not exclude patients undergoing immunotherapy. Technical aspects affecting the results may also be important. In the Italian study, $405 \mathrm{~nm}$ light was used to measure absorbance, while in our trial wavelength was $412 \mathrm{~nm}$. The dilution of samples could also be different; however, to the extent permitted by the manufacturer of the assay kit.

Irrespective of the presented explanations, the results of our study confirming the participation of PAF-AH in the pathogenesis of anaphylaxis lead to the consideration of the practical application of the measurements of plasma PAF-AH. In our population, all patients with a history of anaphylaxis had decreased PAF-AH activity. This confirms that the determination of PAF-AH activity can be a particularly useful tool in the stratification of the individual risk of anaphylaxis toHymenoptera venoms, especially in beekeepers. Assuming that the clinical effect of stinging, on the one hand, depends on the strength of the anaphylactic effect of the venom, and on the other hand on the individual properties of the sensitized person, the measurement of PAF-AH plasma activity allows us to assess a significant internal factor influencing the occurrence of symptoms. Perhaps the test could also help in the diagnosis of non-allergic and idiopathic anaphylaxis distinguishing the true anaphylaxis from numerous conditions with similar symptoms, such as somatoform disorders. Presently, the only laboratory test widely used in such cases is the measurement of tryptase concentration during an acute episode, which is often impossible to perform for obvious reasons. The measurement of plasma PAF-AH activity could provide a lot of diagnostic information, even months or years after an anaphylaxis episode.

Unfortunately, our study plan did not take into account the group of patients with other severe forms of hypersensitivity without a history of an anaphylactic reaction. This would allow us to answer whether the decreased PAF-AH activity occurs only in patients with anaphylaxis, or in general in patients with severe hypersensitivity to one or more factors.

A relatively wide dispersion of the results of plasma PAF-AH activity was found in the control group. Noteworthy, this group was selected primarily on the basis of negative atopic history. It is impossible to predict a priori whether some of these people will be at risk of developing anaphylaxis in the future. Perhaps currently healthy individuals with low PAF-AH activity could be considered capable of developing an anaphylactic reaction in the future. The distribution of results in the control group may also suggest the presence of PAF-AH gene polymorphisms in the Polish population, which strongly affect the enzyme activity. These problems will be the subject of further research. 


\section{5 | CONCLUSIONS}

- The plasma activity of PAF-AH is a promising parameter that can help to distinguish a group of patients not threatened with development of anaphylaxis and not requiring laborious or expensive prophylactic procedures.

- Reduced plasma PAF-AH activity below $17 \mathrm{nmol} / \mathrm{min} / \mathrm{ml}$ is characteristic of patients who develop anaphylactic reactions after an insect sting.

- Contrary to expectations, no significant correlation was found between the degree of impairment of plasma PAF-AH activity and the degree of severity of anaphylaxis following an insect sting.

\section{6 | REFERENCES}

1. Benveniste J, Henson PM, Cochrane CG. Leukocyte-dependent histamine release from rabbit platelets. The role of IgE, basophils, and a platelet-activating factor. J Exp Med . 1972;136(6):1356-77.

2. Wang M, Shibamoto T, Tanida M, et al. Mouse anaphylactic shock is caused by reduced cardiac output, but not by systemic vasodilatation or pulmonary vasoconstriction, via PAF and histamine. Life Sci . 2014;116(2):98-105.

3. Vadas P, Perelman B, Liss G. Platelet-activating factor, histamine, and tryptase levels in human anaphylaxis. J Allergy Clin Immunol . 2013;131(1):144-9.

4. Farr RS, Cox CP, Wardlow ML, et al. Preliminary studies of an acid-labile factor (ALF) in human sera that inactivates platelet-activating factor (PAF). Clin Immunol Immunopathol . 1980;15(3):318-30.

5. Stafforini DM, McIntyre TM, Carter ME et al. Human plasma platelet-activating factor acetylhydrolase. Association with lipoprotein particles and role in the degradation of platelet-activating factor. $J$ Biol Chem . 1987;262(9):4215-22.

6. McIntyre TM, Prescott SM, Stafforini DM. The emerging roles of PAF acetylhydrolase. J Lipid Res . 2009;50 Suppl: S255-9.

7. Thompson A, Gao P, Orfei L, et al. Lipoprotein-associated phospholipase $\mathrm{A}_{(2)}$ and risk of coronary disease, stroke, and mortality: collaborative analysis of 32 prospective studies. Lancet . 2010;375(9725):1536-44.

8. Vadas P, Gold M, Liss G et al. PAF acetylhydrolase predisposes to fatal anaphylaxis. J Allergy Clin Immunol . 2003;111(S206).

9. Vadas P, Gold M, Perelman B et al. Platelet-activating factor, PAF acetylhydrolase, and severe anaphylaxis. N Engl J Med . 2008;358(1):28-35.

10. Pravettoni V, Piantanida M, Primavesi L, et al. Basal platelet-activating factor acetylhydrolase: prognostic marker of severe Hymenoptera venom anaphylaxis. J Allergy Clin Immunol . 2014;133(4):121820.

11. Mueller HL. Diagnosis and treatment of insect sensitivity. J Asthma Res . 1966;3(4):331-3.

12. World Medical Association. World Medical Association Declaration of Helsinki Ethical Principles for Medical Research Involving Human Subjects. JAMA . 2013;310 (20), 2191-2194.

13. Florkowski CM. Sensitivity, Specificity, Receiver-Operating Characteristic (ROC) Curves and Likelihood Ratios: Communicating the Performance of Diagnostic Tests. Clin Biochem Rev . 2008 Aug; 29(Suppl 1): S83-S87.

14. Perelman B, Adil A, Vadas P. Relationship between platelet activating factor acetylhydrolase activity and apolipoprotein B levels in patients with peanut allergy. Allergy Asthma Clin Immunol.2014;10(1):20.

15. Esakova N, Saakyan E, Kondratieva N i wsp.. Platelet-Activating Factor-Acetylhydrolase Gene (PLA2G7) Expression in Children with a History of Food Anaphylaxis. J Allergy Ther . 2015;6(211).

Table 1. Characteristics of the study subgroups - continuous variables.

\begin{tabular}{|c|c|c|c|c|c|}
\hline Variable Subgrc & Ipubgr & pubgr & pubgre & pSubgro & |psubgroup \\
\hline $\begin{array}{l}\text { I-II } \mathbf{n} \\
=39\end{array}$ & $\begin{array}{l}\text { I-II n } \\
=39\end{array}$ & $\begin{array}{l}\text { I-II n } \\
=39\end{array}$ & $\begin{array}{l}\text { III-IV } \\
\mathrm{n}= \\
50\end{array}$ & $\begin{array}{l}\text { III-IV } \\
\mathrm{n}= \\
50\end{array}$ & $\begin{array}{l}\text { III-IV } \\
\mathrm{n}= \\
50\end{array}$ \\
\hline
\end{tabular}




\begin{tabular}{llllllllllll} 
& Mean & SD & Median & Mean & SD & Median & & Mean & SD & Median & vs $\mathbf{1}$ \\
$\begin{array}{l}\text { Age } \\
\text { [years] }\end{array}$ & $\mathbf{4 8 , 2 6}$ & 12,20 & 46,00 & $\mathbf{4 5 , 3 6}$ & 13,88 & 43,50 & 0,2301 & $\mathbf{4 4 , 1 5}$ & 13,33 & 45,00 & 0,2810 \\
$\begin{array}{l}\text { Body } \\
\text { mass }\end{array}$ & $\mathbf{8 1 , 9 5}$ & 17,99 & 85,00 & $\mathbf{7 6 , 5 6}$ & 15,16 & 75,00 & 0,1160 & $\mathbf{7 9 , 5 0}$ & 12,74 & 80,00 & 0,6637 \\
$\begin{array}{l}\text { [kg] } \\
\text { Height } \\
{[\mathbf{c m}]}\end{array}$ & $\mathbf{1 7 1 , 8 6}$ & 9,86 & 171,00 & $\mathbf{1 7 1 , 0 2}$ & 9,09 & 170,00 & 0,5700 & $\mathbf{1 7 5 , 4 4}$ & 11,49 & 174,50 & 0,3528 \\
$\begin{array}{l}\mathbf{B M I} \\
{\left[\mathrm{kg} / \mathbf{m}^{\mathbf{2}}\right]}\end{array}$ & $\mathbf{2 7 , 5 9}$ & 5,03 & 27,14 & $\mathbf{2 6 , 0 5}$ & 4,01 & 25,46 & 0,1002 & $\mathbf{2 5 , 8 4}$ & 3,29 & 26,44 & 0,3023 \\
$\begin{array}{l}\text { Tryptase 5,59 } \\
{[\mathbf{n g} / \mathbf{m l}]}\end{array}$ & 4,13 & 3,94 & $\mathbf{4 , 7 7}$ & 2,69 & 4,04 & 0,8167 & $\mathbf{5 , 4 6}$ & 2,53 & 4,89 & 0,2878 \\
\hline
\end{tabular}

Tab. 2. Comparison of subgroups within the study population regarding plasma PAF acetylhydrolase activity.

\begin{tabular}{|c|c|c|c|c|c|}
\hline $\begin{array}{l}\text { Comparised } \\
\text { series }\end{array}$ & $\begin{array}{l}\text { Mean } \\
{[\mathrm{nmol} / \mathrm{min} / \mathrm{ml}]}\end{array}$ & SD & Median & Test & $\mathbf{p}$ \\
\hline $\begin{array}{l}\text { Control } \\
\text { group }\end{array}$ & 21,38 & 9,50 & 25,59 & $\begin{array}{l}\text { U Manna- } \\
\text { Whitney }\end{array}$ & 0,000001 \\
\hline $\begin{array}{l}\text { Subgroup } \\
\text { I-II }\end{array}$ & 9,47 & 1,87 & 9,15 & & \\
\hline $\begin{array}{l}\text { Control } \\
\text { group }\end{array}$ & 21,38 & 9,50 & 25,59 & $\begin{array}{l}\text { U Manna- } \\
\text { Whitney }\end{array}$ & 0,000014 \\
\hline $\begin{array}{l}\text { Subgroup } \\
\text { III-IV }\end{array}$ & 10,16 & 2,50 & 9,85 & & \\
\hline $\begin{array}{l}\text { Subgroup } \\
\text { I-II }\end{array}$ & 9,47 & 1,87 & 9,15 & t Student & 0,060902 \\
\hline $\begin{array}{l}\text { Subgroup } \\
\text { III-IV }\end{array}$ & 10,16 & 2,50 & 9,85 & & \\
\hline $\begin{array}{l}\text { Control } \\
\text { group }\end{array}$ & 21,38 & 9,50 & 25,59 & $\begin{array}{l}\text { Kruskal-Wallis } \\
\text { test }\end{array}$ & 0,0001 \\
\hline $\begin{array}{l}\text { 1st degree of } \\
\text { severity }\end{array}$ & 8,93 & 2,13 & 8,97 & & \\
\hline $\begin{array}{l}\text { 2nd degree } \\
\text { of severity }\end{array}$ & 9,61 & 1,80 & 9,23 & & \\
\hline $\begin{array}{l}\text { 3rd degree } \\
\text { of severity }\end{array}$ & 10,24 & 2,64 & 9,67 & & \\
\hline $\begin{array}{l}\text { 4th degree } \\
\text { of severity }\end{array}$ & 10,01 & 2,26 & 9,94 & & \\
\hline
\end{tabular}

Figure 1. Scatter plot of PAF-AH plasma activity by severity of anaphylaxis. 


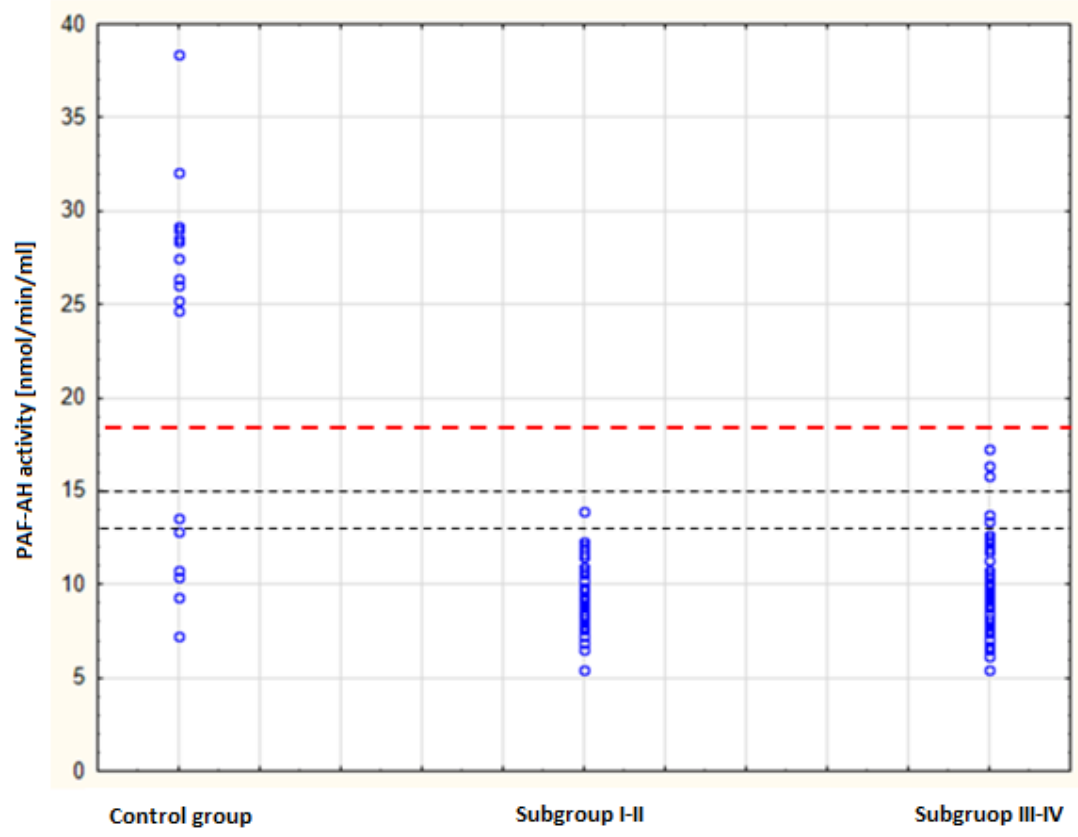

Figure 2. Distribution of PAF-AH plasma activity by severity of anaphylaxis - box plot. 0 - control group, $1,2,3,4$ - grade of severity according to Müller's classification.

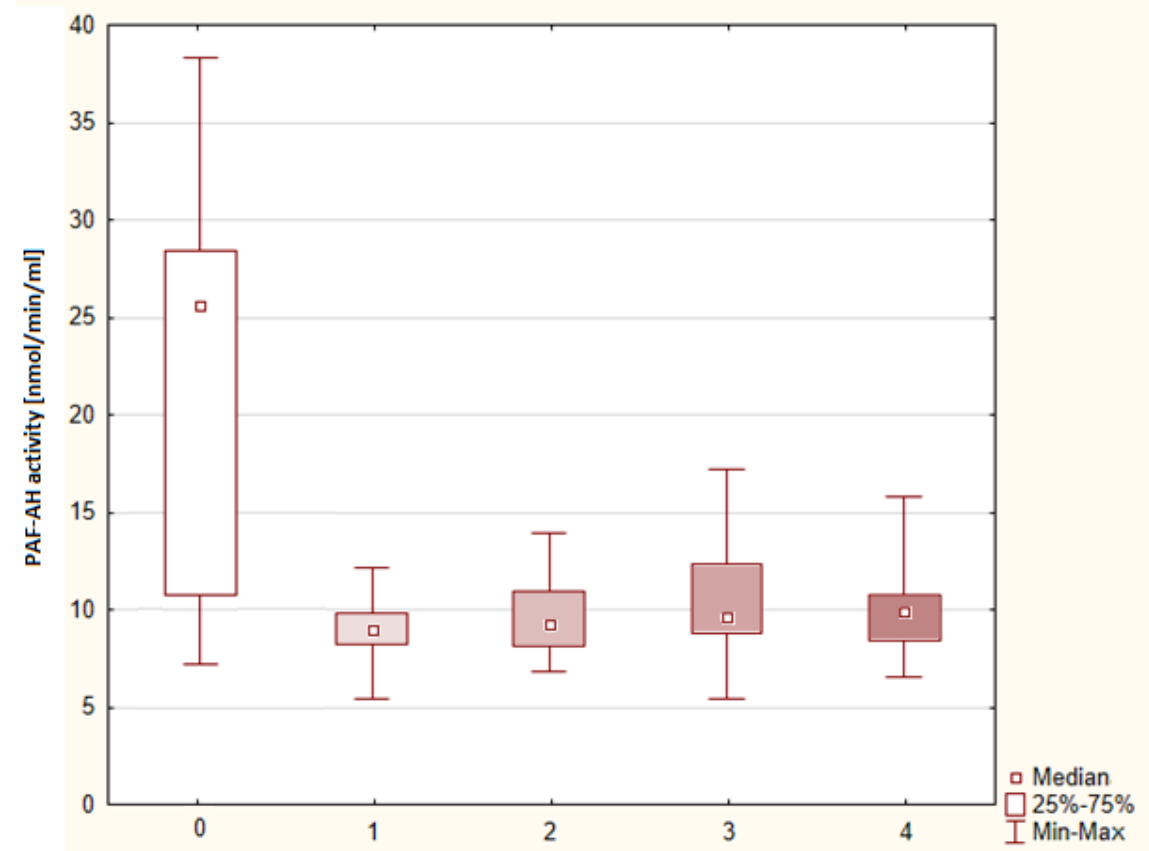

Figure 3. ROC for PAF-AH plasma activity test. 


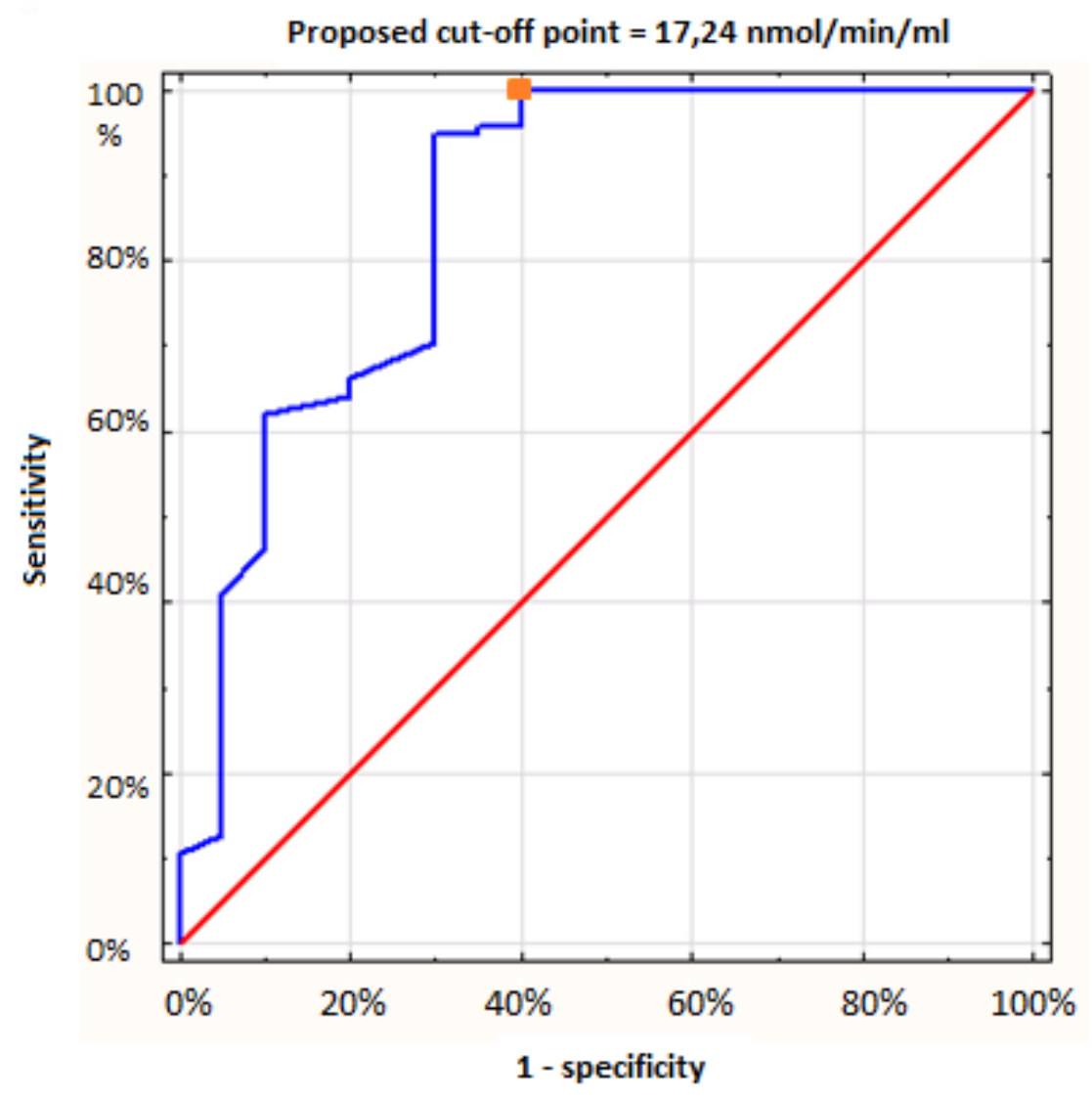

